\title{
Structural Characterization of Emerging Pathogenic Human Parvoviruses
}

Mario Mietzsch ${ }^{1}$, Mengxiao Luo ${ }^{1}$, Jennifer C. Yu ${ }^{1}$, Shweta Kailasan ${ }^{1}$, Maria Ilyas ${ }^{1}$, Paul Chipman ${ }^{1}$, Duncan Sousa $^{2}$, Maria Söderlund-Venermo ${ }^{3}$, Robert McKenna ${ }^{1}$, and Mavis Agbandje-McKenna ${ }^{1}$

1. Department of Biochemistry and Molecular Biology, Center for Structural Biology, McKnight Brain Institute, College of Medicine, University of Florida, Gainesville, FL, USA

2. Biological Science Imaging Resource, Department of Biological Sciences, The Florida State University, Tallahassee, FL, USA

3. Department of Virology, University of Helsinki, Helsinki, Finland

Parvoviruses are small ssDNA viruses with a non-enveloped capsid. Members of the family Parvoviridae are divided into the subfamilies Densovirinae, infecting invertebrates, and Parvovirinae that utilize vertebrates as hosts. In 1974 Parvovirus B19 was discovered and was the only parvovirus known to be pathogenic in humans for more than three decades. Recent advances in DNA sequencing technology have led to the discovery of several new parvoviruses capable of infecting humans, including human bocaparvoviruses 1 to 4 (HBoV1-HBoV4), bufaviruses 1 to 3 (BuV1-BuV3), cutavirus $(\mathrm{CuV})$, tusavirus $(\mathrm{TuV})$, and human parvovirus 4 (PARV4). These viruses were found in different tissues, including the gastro-intestinal tract, the respiratory tract, erythroid progenitor cells, and cutaneous malignant melanoma lesions. The viral capsid plays a central role in the infection of these cells since it mediates the attachment of the virus to specific receptors on the target cells. After cell entry the capsid remains intact while it travels through the endo/lysosomal pathway to the nucleus where the viral genome is released. At the end of the replication cycle newly generated capsids package newly replicated genomes and protect these for another round of replication.

We have determined the capsid structures for several of these emerging viruses to assist in functional characterization and the development of strategies to control virus infection. The structures of HBoV1HBoV4, BuV1-3, TuV, and $\mathrm{CuV}$ by cryo-electron microscopy and image reconstruction (cryo-EM) in the resolution range of 2.6 to $3.0 \AA$. The resolution of the maps enabled the atomic assignment of the amino acids of the major capsid protein, VP3 (Fig. 1). These viruses, which display different tissue tropisms and sequence identities as low as 14.9\% (e.g. BuV2 vs. B19), share common capsid features with other parvoviruses including depressions at the icosahedral 2-fold and surrounding the 5-fold symmetry axes, protrusions at or surrounding the 3-fold axes, and a channel at the 5-fold axes (Fig. 2). Furthermore, the overall VP2 structure topology of these viruses are highly similar, with the core being completely superposable. The differences are localized to surface loops, in previously defined common variable regions, which have been shown to be involved in receptor binding, cellular trafficking, transcription, and antigenic reactivity. In addition, the human bocaviruses display the presence of a density ("basket") beneath the 5-fold axis that extends the 5-fold channel into the capsid interior. This density is conserved among the bocaparvoviruses and suggestive of a genus specific function. In contrast, the bufaviruses, $\mathrm{CuV}$, and $\mathrm{TuV}$ display the most outwardly extended 5-fold channels among the parvoviruses (Fig. 2). These structures provide a 3D platform for functional annotation of these pathogens that will help to understand their disease mechanisms on a molecular level towards the development of therapeutics. 
References:

[1] Mietzsch et al., J Virol , 91, (2017) e00261-17

[2] Ilyas et al., Viruses, 10 (2018) 22.

[3] Kaufmann et al., PNAS, 101 (2004) p. 11628.

[4] Data collection at FSU was made possible by NIH grants S10 OD018142-01, purchase of a direct electron camera for the Titan-Krios at FSU (PI Taylor), S10 RR025080-01, purchase of a FEI Titan Krios for 3-D EM (PI Taylor), and U24 GM116788, the Southeastern Consortium for Microscopy of MacroMolecular Machines (PI Taylor).

[5] The University of Florida COM and NIH GM082946 (to MAM and RM) provided funds for the research efforts at the University of Florida.

[6] The Sigrid Jusélius Foundation provided funds for the research efforts at the University of Helsinki, Finland.

[7] We thank Dr. Hong Zhou (University of California Los Angeles) and the NIH "West/Midwest Consortium for High-Resolution Cryo Electron Microscopy" project for access to the Electron Imaging Center for Nanomachines's Titan Krios and K2 DED utilized for high-resolution data collection

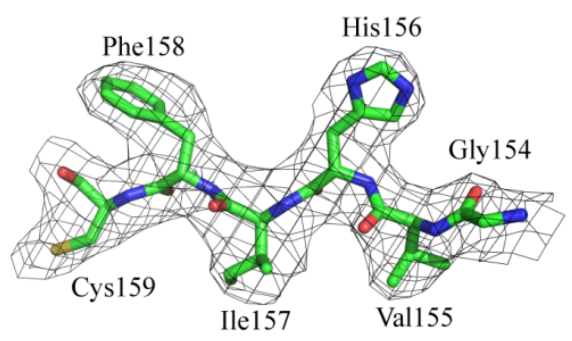

HBoV1 $(2.87 \AA)$

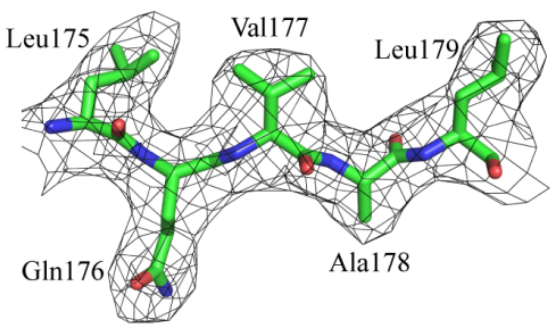

$\operatorname{BuV1}(2.84 \AA)$

Figure 1. Density maps and atomic models for HBoV1 and BuV1. The amino acid residues are shown as stick representation and colored according to atom type: $\mathrm{C}=$ green, $\mathrm{O}=\mathrm{red}, \mathrm{N}=$ blue inside a black mesh density map. This image was generated using PyMOL.
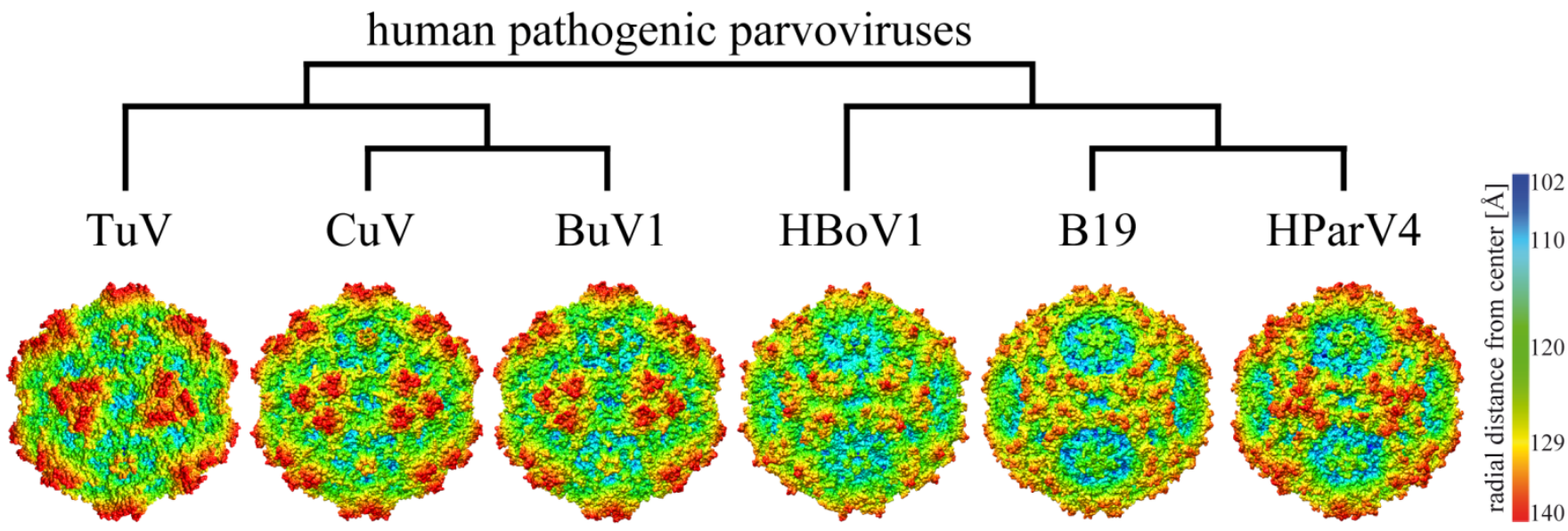

Figure 2. Cladogram showing the relationship between the pathogenic human parvoviruses. This image was generated online (http://www.phylogeny.fr/) utilizing the VP2 sequences as input. Radial-colored capsid surface representations (blue to red) are viewed along the 2-fold axis and generated using Chimera. The capsid surface image for HParv4 was generated from model predictions. 